\title{
On sojourn times, excursions and spectral measures connected with quasidiffusions
}

By

\author{
Uwe KüCHLER
}

\section{Introduction.}

Let $X=\left(X_{t}\right)_{t \geq 0}$ be a quasidiffusion on the real line with natural scale and speed measure $m$, i.e. a strong Markov process the infinitesimal generator of which is a restriction of the generalized second order differential operator $D_{m} D_{x}$.

Examples are diffusions and birth-and death-processes. We shall consider excursions of $X$ from 0 over some level $a>0$ and study the sojourn time $T$ which $X$ spends over $a^{\prime}\left(a^{\prime} \in[0, a]\right)$ during such an excursion.

If $a^{\prime}=a$, then $T$ has a mixed exponential distribution. The mixing measure can be identified with the normalized spectral-measure of a so-called dual string (see Proposition 3.2).

We consider the process $\left(P_{t}\left(a, a^{\prime}\right)\right)_{t \geq 0}$ where $P_{t}\left(a, a^{\prime}\right)$ is equal to the time which $X$ spends over $a^{\prime}$ during excursions of $X$ from 0 to $a$ occuring before the local time $l(\cdot, 0)$ of $X$ at zero equals $t$. If $a^{\prime}>0$ it turns out to be a randomly stopped compounded Poisson process (Theorem 4.4).

If $a^{\prime}=0$ or $a^{\prime}=a$ its Laplace transform can be calculated by the spectral measures of some corresponding dual strings (see point 4.2 below). In parituclar, if $\nu$ denotes the Ito excursion law of $X$ at zero, $V$ the "peak" of an excursion, we obtain the Laplace transform of the measure $\nu(T \in d t, V \geqq a)$ (Corollary 4.6). Finally a connection between the trajectories of $X$ and some spectral measures via the local time of $X$ is derived (Proposition 4.10). This extends a result of Ito, McKean [5] for diffusions.

\section{Quasidiffusions, strings and spectral measures.}

In this chapter we summarize some definitions and facts on quasidiffusions and related topics which are necessary in the sequel. Proofs are omitted, details can be found e.g. in $[2,3,5,6,9-13]$ or can be easily derived from those.

We denote by $R$ the real line, by $\mathfrak{B}$ the $\sigma$-algebra of its Borelian subsets and by $K$ the set of complex numbers. Let $b \mathfrak{B}$ be the set of all bounded measurable real functions on $R$. 


\subsection{Quasidiffusions.}

Let $m$ be an extended real-valued right-continuous nondecresaing function on $R$ and put

$$
\begin{aligned}
& l_{0}:=\inf \{x \in R \mid m(x)>-\infty\}, \quad l_{1}:=\sup \{x \in R \mid m(x)<\infty\}, \\
& r_{0}:=\inf \left\{x>l_{0} \mid m(x)>m\left(l_{0}\right)\right\}, \quad r_{1}:=\sup \left\{x<l_{1} \mid m(x)<m\left(\left(l_{1}-0\right)\right\},\right. \\
& m_{0}:=m(\{0\}) .
\end{aligned}
$$

Assume

$$
m(0-0)=0, \quad m(x) \neq 0, \quad l_{0}<0<r_{1}
$$

and define

$$
I:=\left(l_{0}, l_{1}\right), E:=\left[r_{0}, r_{1}\right] \cap I .
$$

Denote by $E_{m}$ the points of $E$ in which $m$ increases. The function $m$ and the measure generated by $m$ on $I$ are identified.

Let $W=\left(W_{t}, \mathscr{F}_{t}^{W},\left(P_{x}\right)_{x \in R}\right)$ be a standard Wiener process and $l^{W}(t, x)(t \geqq 0, x \in R)$ its (continuous in $(t, x)$ ) local time with

$$
\int_{R} l(t, x) f(x) d x=\int_{0}^{t} f\left(W_{s}\right) d s \quad(t \geqq 0, f \in b \mathfrak{B}) .
$$

Put

$$
\begin{aligned}
S_{t}:=\int_{R} l^{W}(t, x) m(d x) & (t \geqq 0) \\
T_{t}:=\inf \left\{u \geq 0 \mid S_{u}>t\right\} & (t \geqq 0)
\end{aligned}
$$

with the definition inf $\phi:=\infty$.

Then $\left(T_{t}\right)_{t \leq 0}$ is a right-continuous strictly increasng process and $T_{t}$ is an $\mathscr{F}^{W}$-stopping time $\left(\mathscr{F}^{W}:=\left(\mathscr{F}_{t}^{W}\right)_{t \geqq 0}\right)$. Define

$$
\begin{aligned}
& \mathscr{F}_{t}:=\mathscr{F}_{T_{t}}^{W} \quad(t \geqq 0), \\
& \zeta:=\inf \left\{t>0 \mid W_{T_{t}} \in\left(l_{0}, l_{1}\right)\right\}, \\
& X_{t}:=W_{T_{t}} \quad(0 \leqq t<\zeta) .
\end{aligned}
$$

Then $X=\left(X_{t}, \zeta, \mathscr{F}_{t},\left(P_{x}\right)_{x \in E_{m}}\right)$ is a right-continuous strong Markov process with state space $E_{m}$ killed at the time $\zeta$. We call $X$ the quasidiffusion with speed measure $m$ (other notations are: generalized diffusion process [10], gap diffusion [9]). $X$ is skip free in the sense that

$$
\left(X_{t} \wedge X_{t-}, X_{t} \vee X_{t-}\right) \cap E_{m}=\emptyset \quad(0<t<\zeta) .
$$

In particular, starting at $x<y \in E_{m}, X$ enters $\left[y, l_{1}\right]$ through $y$ : we have with $\sigma_{y}:=\inf \left\{t>0 \mid X_{t} \geqq y\right\}$ the equality 


$$
X_{\sigma_{y}}=y \quad \text { on } \quad\left\{\sigma_{y}<\infty\right\} \bmod P_{x} .
$$

The analogous property holds if $x>y$.

If $m$ is strictly increasing, it follows that $E_{m}=E$ and $X$ is continuous, i.e. $X$ is a diffusion in the sense of [5]. If $m$ is piecewise constant and $E_{m}$ has no point of accumulation in $\left(r_{0}, r_{1}\right)$, then $X$ is a birth- and death-process.

By

$$
\begin{array}{ll}
l(t, x):=l^{W}\left(T_{t}, x\right) & (0 \leqq t<\zeta) \text { and } \\
l(\zeta, x):=l(\zeta-0, x) & \left(x \in E_{m}\right)
\end{array}
$$

the local time $l$ of $X$ is defined. It is continuous in both variables and for every bounded measurable real function $f$ on $E_{m}$ we have

$$
\int_{E_{m}} f(x) l(t, x) m(d x)=\int_{0}^{t} f\left(X_{s}\right) d s \quad(0 \leqq t<\zeta)
$$

in particular

$$
m_{0} \cdot l(t, 0)=\int_{0}^{t} \mathbf{1}_{\{0\}}\left(X_{s}\right) d s \quad(0 \leqq t<\zeta)
$$

Put

$$
\tau_{y}^{W}:=\inf \left\{t>0 \mid W_{t}=y\right\}, \quad \tau_{y}:=\inf \left\{t>0 \mid X_{t}=y\right\} \quad\left(y \in E_{m}\right) .
$$

Then it holds

$$
T_{\tau_{y}}=\tau_{y}^{W}\left(y \in E_{m}\right), \quad T_{\zeta}=\tau_{l_{0}}^{W} \wedge \tau_{l_{1}}^{W}=: \zeta^{W} .
$$

Using (1) with $f \equiv 1$ we obtain

$$
\tau_{y}=\int_{E_{m}} l\left(\tau_{y}, x\right) m(d x)=\int_{E_{m}} l^{W}\left(\tau_{y}^{W}, x\right) m(d x) \quad\left(y \in E_{m}\right) .
$$

If $G \in \mathfrak{B}$, we define a measure $m^{G}$ on $I$ by $d m^{G}:=\mathbf{1}_{G} d m$ and mark all quantities connected with $m^{G}$ by $G$, e.g. $X^{G}, \tau_{y}^{G}$. From (1) and (3) we get

$$
\tau_{y}^{G}=\int_{0}^{\tau_{y}} \mathbf{1}_{G}\left(X_{s}\right) d s \quad\left(y \in E_{m} \cap G\right) .
$$

With $m$ there is connected a generalized second order differential operator $D_{m} D_{x}$ defined by ${ }^{1)}$

$$
D_{m} D_{x} f=g: \Leftrightarrow f(x)=f(0)+x D^{-} f(0)+\int_{0}^{x}(x-s) g(s) m(d s), \quad(x \in I)
$$

on an appropriately chosen domain of continuous functions on $I$ which are linear on the components of the open set $I \backslash E_{m}$. The restriction of $D_{m} D_{x}$ to the Banach space $\boldsymbol{C}_{m}$ of all such functions that are bounded and satisfy

1) $D^{-}\left(D^{+}\right)$denotes the left (right) hand side derivation operator. 


$$
\lim _{x \rightarrow l_{i}} f(x)=0 \quad \text { if } \quad\left|l_{i}\right|<\infty \quad(i=0,1)
$$

is the infinitesimal generator of $X$.

Let $\varphi$ and $\psi$ be the (uniquely determined) solution of

$$
\begin{aligned}
& \varphi(x, \lambda)=1-\lambda \int_{0}^{x}(x-s) \varphi(s, \lambda) m(d s) \quad \text { and } \\
& \psi(x, \lambda)=x-\lambda \int_{0}^{x}(x-s) \psi(s, \lambda) m(d s), \quad(\lambda \in K, x \in I)
\end{aligned}
$$

respectively.

It holds the Lagrange identity, i.e. for every $\lambda \in K$ we have

$$
\varphi(x, \lambda) D^{-} \psi(x, \lambda)-\psi(x, \lambda) D^{-} \varphi(x, \lambda) \equiv 1 \quad\left(x \in E_{m}\right) .
$$

Suppose $z \in E_{m} \cup\left(l_{0}, l_{1}\right), \lambda \in K_{0}:=K \backslash[0, \infty)$ and put

$$
\begin{array}{ll}
\chi_{+}^{2}(x, \lambda):=\varphi(x, \lambda) \int_{x}^{z} \varphi^{-2}(s, \lambda) d s & \left(z \geqq 0, l_{0} \leqq x \leqq z\right) \\
\chi_{-}^{2}(x, \lambda):=\varphi(x, \lambda) \int_{z}^{x} \varphi^{-2}(s, \lambda) d s & \left(z \leqq 0, z \leqq x \leqq l_{1}\right) .
\end{array}
$$

If $z=l_{0}$ or $=l_{1}$ we omit the mark $z: \chi_{-}^{l_{0}}=\chi_{-}, \chi_{+}^{l_{1}}=\chi_{+}$. Let $z_{0}, z_{1}$ be given with $z_{0} \leqq 0, z_{1}>0, z_{1} \in E_{m} \cup\left(l_{0}, l_{1}\right)(i=0,1)$. Then, for $x \in E_{m} \cap\left(z_{0}, z_{1}\right) \sigma:=\zeta \wedge \inf \{t>0 \mid$ $\left.X_{i} \in\left(z_{0}, z_{1}\right)\right\}, \lambda<0$, we have for any $f \in b \mathfrak{B}$

$$
E_{x} \int_{0}^{\sigma} e^{\lambda t} f\left(X_{t}\right) d t=\int_{z_{0}}^{z_{1}} \chi_{-}^{z_{0}}(x \wedge y, \lambda) \chi_{+}^{z_{1}}(x \vee y, \lambda) f(y) m(d y) .
$$

From this formula, the strong Markov property, and the skip freeness of $X$ it follows under the same assumptions on $z_{0}, z_{1}, x$ and $\lambda$ by a known method of [5]:

$$
\begin{aligned}
& E_{x}\left[\exp \left(\lambda \tau_{y}\right) \cdot \mathbf{1}_{\left\{\tau_{z_{1}}>\tau_{y}\right\}}\right]=\frac{\chi_{+}^{z_{1}(x, \lambda)}}{\chi_{+}^{z_{1}(y, \lambda)}} \quad\left(y \in E_{m}, y \leqq x \leqq z_{1}\right), \\
& E_{x}\left[\exp \left(\lambda \tau_{y}\right) \cdot \mathbf{1}_{\left\{\tau_{z_{0}}>\tau_{y}\right\}}\right]=\frac{\chi_{-}^{z_{0}}(x, \lambda)}{\chi_{-}^{z_{0}}(y, \lambda)} \quad\left(y \in E_{m}, z_{0} \leqq x \leqq y\right) .
\end{aligned}
$$

\subsection{Strings and dual strings.}

A measure $m$ as considered above is called a string if $l_{0}=-\infty$ and $r_{0} \geqq 0$. Let $\mathfrak{M}$ be the set of all strings and assume $m \in \mathfrak{M}$. The function $h$ defined by

$$
h(\lambda):=\int_{0}^{l_{1}} \varphi^{-2}(x, \lambda) d x \quad\left(\lambda \in K_{0}\right)
$$

is called the characteristic function of $m$ ([10]). We have

$$
h(\lambda)=\chi_{+}(0, \lambda)=\lim _{x_{\uparrow l_{1}}} \frac{\psi(x, \lambda)}{\varphi(x, \lambda)} \quad \text { and }
$$


Sojourn times, excursions and spectral measures

$$
\chi_{+}(x, \lambda)=h(\lambda) \varphi(x, \lambda)-\psi(x, \lambda) \quad\left(\lambda \in K_{0}\right)
$$

If $m, m^{\prime} \in \mathfrak{M}, a, b, m_{0}^{\prime} \geqq 0, c>0$ and

$$
m^{\prime}(x)=m_{0}^{\prime}+\frac{b}{c} m\left(\frac{x-a}{c}\right), \quad(x \in R)
$$

then the characteristic functions $h_{m}$ and $h_{m^{\prime}}$ of $m$ and $m^{\prime}$, respectively, are related by

$$
\frac{1}{h_{m^{\prime}}(\lambda)}=-\lambda m_{0}^{\prime}+\frac{1}{a+c h_{m}(b \lambda)} \quad\left(\lambda \in K_{0}\right) \text {. }
$$

For every $m \in \mathfrak{M}$ there exists a $\sigma$-finite measure $\sigma$ on $[0, \infty)$, called the spectral measure of $m$, with

$$
\int_{[0, \infty)} \frac{\sigma(d u)}{1+u}<\infty
$$

such that

$$
\begin{array}{ll}
h(\lambda)=r_{0}+\int_{[0, \infty)} \frac{\sigma(d u)}{u-\lambda} & \left(\lambda \in K_{0}\right), \\
\sigma([0, u))=\frac{1}{\pi} \lim _{c \downarrow 0} \int_{[0, u)} \operatorname{Im} h(b+i c) d b & (u \geqq 0) .
\end{array}
$$

Moreover, we have

$$
\begin{aligned}
& \sigma([0, \infty))=\frac{1}{m_{0}} \text { and } \\
& \sigma(\{0\})=\frac{1}{m\left(r_{1}\right)} \text { if } l_{1}=\infty,=0 \text { if } l<\infty .
\end{aligned}
$$

If $m$ is a measure as in 2.1. above and $x \in E_{m}$, then two strings $m_{x}^{+}$are given by

$$
\begin{aligned}
& m_{x}^{-}([0, y)):=m((x-y, x]), \\
& m_{x}^{+}([0, y)):=m([x, x+y)) \quad(y>0) .
\end{aligned}
$$

Note that $m_{x}^{-}(\{0\})=0, m_{x}^{+}(\{0\})=m(\{x\})$.

For the characteristic functions $h_{x}^{+}$of $m_{x}^{-}$, respectively, we obtain after some calculations

$$
h_{x}^{+}(\lambda)=\frac{\chi_{-}(x, \lambda)}{D^{-} \chi_{-}(x, \lambda)}, \quad h_{x}^{+}(\lambda)=-\frac{\chi_{+}(x, \lambda)}{D^{-} \chi_{+}(x, \lambda)} \quad\left(\lambda \in K_{0}\right),
$$

If $G=[a, \infty)(a>0),(9)$ and (14) imply

$$
h_{0}^{G,+}(\lambda)=h_{a}^{+}(\lambda)+a=\frac{a D^{-} \chi_{+}(a, \lambda)-\chi_{+}(a, \lambda)}{D^{-} \chi_{+}(a, \lambda)} \quad\left(\lambda \in K_{0}\right) .
$$


Let $m \in \mathfrak{M}$ and put

$$
m_{d}(x):=\inf \{y>0 \mid m(y)>x\} \quad(x \geqq 0),=0 \quad(x>0) .
$$

Then it holds that $m_{d} \in \mathfrak{M}$ and $\left(m_{d}\right)_{d}=m$. The string $m_{d}$ is called the dual string to $m$. All quantities connected with $m_{d}$ will be marked by $d$, e.g. $\psi_{d}, \varphi_{d}, \sigma_{d}$. With the notation

$$
x_{+}:=\inf \left\{y>x \mid y \in E_{m} \cup\left\{l_{1}\right\}\right\}, \quad x_{-}=\sup \left\{y<x \mid y \in E_{m} \cup\left\{l_{0}\right\}\right\} \quad\left(x \in E_{m}\right)
$$

we get for $x \in I, \lambda \in K$

$$
\begin{gathered}
-\lambda \psi(x, \lambda)=\left.D^{+} \varphi_{d}(z, \lambda)\right|_{z=m(x)}+\lambda\left(x_{+}-x\right) \varphi_{d}(m(x), \lambda) \\
\varphi(x, \lambda)=\left.D^{+} \psi_{d}(z, \lambda)\right|_{z=m(x)}+\lambda\left(x_{+}-x\right) \psi_{d}(m(x), \lambda) .
\end{gathered}
$$

These equations provide

$$
h_{d}(\lambda)=-(\lambda h(\lambda))^{-1} \quad\left(\lambda \in K_{0}\right) .
$$

We conclude this point with several formulas concerning the dual strings $m_{d}$. From (10) and (13) it follows

$$
h_{d}(\lambda)=m_{0}-\left(\lambda l_{1}\right)^{-1}+\int_{(0, \infty)} \frac{\sigma_{d}(d u)}{u-\lambda} \quad\left(\lambda \in K_{0}\right) .
$$

Thus we have by (14)

$$
\begin{aligned}
& l_{1}^{-1}-h^{-1}(\lambda)=m_{0}+\int_{(0, \infty)}\left(\frac{1}{u}-\frac{1}{u-\lambda}\right) u \sigma_{d}(d u)= \\
= & m_{0}+\int_{(0, \infty)}\left(1-e^{\lambda u}\right) \vartheta_{d}(u) d u \quad\left(\lambda \in K_{0}\right)
\end{aligned}
$$

with

$$
\vartheta_{d}(u):=\int_{(0, \infty)} v e^{-u v} \sigma_{d}(d v) . \quad(u>0) .
$$

Note that

$$
\begin{aligned}
\int_{\varepsilon}^{\infty} \vartheta_{d}(u) d u= & \int_{(0, \infty)} e^{-\varepsilon v} \sigma_{d}(d v) \underset{\varepsilon \downarrow 0}{\longrightarrow} \sigma_{d}((0, \infty))=r_{0}^{-1}-l_{1}^{-1} \\
& \left(r_{0}^{-1}:=\infty \text { if } r_{0}=0\right)
\end{aligned}
$$

Furthermore, (14)-(16) imply for $G:=[a, \infty)$

$$
\frac{D^{-} \chi_{+}(a, \lambda)}{\chi_{+}(a, \lambda)-a D^{-} \chi_{+}(a, \lambda)}=\lambda h_{d}^{G}(\lambda) \quad\left(\lambda \in K_{0}, a>0\right)
$$

and using (18) we get for $a>0$ 


$$
\frac{\chi_{+}(a, \lambda)}{\chi_{+}(a, \lambda)-a D^{-} \chi_{+}(a, \lambda)}=1+a \lambda h_{d}^{G}(\lambda)=a \int_{(0, \infty)} \frac{u}{u-\lambda} \sigma_{d}^{G}(d u) \quad\left(\lambda \in K_{0}\right)
$$

( $\sigma_{d}^{G}$ denotes the spectral measure of the string $\left(m^{G}\right)_{d}$ which is dual to $m^{G}$ ).

\subsection{Examples.}

1. If $m(x)=x^{\gamma} \cdot \mathbf{1}_{[0, \infty)}(x)(r>0$ fixed, $x \in R)$, then ' $X$ is a diffusion on $[0, \infty)$ instantaneously reflected at zero. We have

$$
h(\lambda)=\frac{\Gamma\left(\frac{1}{\gamma+1}\right)}{\Gamma\left(\frac{\gamma}{\gamma+1}\right)} \cdot(\gamma+1)^{(1-\gamma) /(1+\gamma)} \cdot(-\lambda \gamma)^{-1 /(\gamma+1)}
$$

and

$$
\sigma(d u)=\left[\Gamma\left(\frac{\gamma}{\gamma+1}\right)\right]^{-2}(\gamma+1)^{(1-\gamma) /(1+\gamma)}(u \gamma)^{-1 /(1+\gamma)} d u \quad(u \geqslant 0)
$$

The dual quantities $m_{d}, h_{d}, \sigma_{d}$ are obtained by substituting $\gamma^{-1}$ for $r$.

2. Put $m(d x):=\left(\mathbf{1}_{[0, v]}(x)+c^{-2} \mathbf{1}_{(v, \infty)}(x)\right) d x(c, v>0$ fixed, $x \in R)$

Then it holds

$$
h(\lambda)=(-\lambda)^{-1 / 2} \cdot \frac{c+\tanh v \sqrt{-\lambda}}{1+c \tanh v \sqrt{-\lambda}} \quad\left(\lambda \in K_{0}\right)
$$

and

$$
\sigma(d u)=\frac{2 c \cdot d u}{\pi u^{1 / 2}\left(1+c^{2}+\left(1-c^{2}\right) \cos 2 v \sqrt{u}\right)} \quad(u>0)
$$

Replacing $c$ by $c^{-1}$ we obtain $m_{d}, h_{d}, \sigma_{d}$.

Define $\quad p(x):=x \mathbf{1}_{[0, v]}(x)+(v+c(x-v)) \mathbf{1}_{[v, \infty)}(x) \quad(x \geqq 0)$, then $\left(p^{-1}\left(X_{t}\right)\right)_{t \geq 0}$ behaves like a skew Brownian motion process reflected at zero with diffusion coefficient 2 and "skew point" $v$.

3. Assume $m$ is a string consisting of a weightless thread carrying beads with mass $m_{i}>0$ at $p_{i}\left(i=1, \cdots, N ; 0<p_{1}<\cdots<p_{N}\right)$. Put $\Delta_{0}:=p_{1}, \quad \Delta_{i}:=p_{i+1}-p_{i}$ $(i=1, \cdots, N-1)$ and $l:=\infty$. It holds

$$
h_{m}(\lambda)=\Delta_{0}+\sum_{k=1}^{N} \frac{\sigma_{k}}{u_{k}-\lambda} \quad\left(\lambda \in K_{0}\right)
$$

where $u_{k}(k=1, \cdots, N)$ are the eigenvalues of the matrix $-A$ which is given by $A=\left(a_{i j}\right), i, j=1, \cdots, N, a_{11}=-\lambda_{1}, a_{i i}=-\left(\lambda_{i}+\mu_{i}\right)(i=2, \cdots, N-1), a_{N N}=-\mu_{N}$, $a_{i i-1}=\mu_{i}(i=2,, \cdots N), a_{i i+1}=\lambda_{i}(i=1, \cdots, N-1), a_{i j}=0$ otherwise, $\lambda_{i}:=\left(m_{i} \cdot \Delta_{i}\right)^{-1}$ $(i=1, \cdots, N-1) \mu_{i}=\left(m_{i} \Delta_{i-1}\right)^{-1}(i=1, \cdots, N)$. Furthermore

$$
\sigma_{k}:=\left(\sum_{i=0}^{N-1} Q_{i}^{2}\left(u_{k}\right) m_{i+1}\right)^{-1},
$$


and $\left(Q_{0}\left(u_{k}\right), \cdots, Q_{N-1}\left(u_{k}\right)\right)^{T}$ is the eigenvector of $-\mathrm{A}$ belonging to $u_{k}$ normalized by $Q_{0}\left(u_{k}\right) \equiv 1,(k=1, \cdots, N)$.

The quasidiffusion $X$ with speed measure $m$ is the birth- and death-process on $p_{1}, \cdots, p_{N}$ with $A$ as its matrix of intensities. The dual string $m_{d}$ has the masses $m_{d, i}=\Delta_{i}(i=0, \cdots, N-1)$ at the points

$$
p_{d, 0}=0, \quad p_{d, k}=\sum_{i=1}^{k} m_{i} \quad(k=1, \cdots, N-1)
$$

and it holds $\left(l_{1}\right)_{d}=\sum_{i=1}^{N} m_{i}$. We have

$$
\begin{aligned}
& h_{d}(\lambda)=\sum_{k=1}^{N} \frac{\sigma_{d, k}}{u_{d, k}-\lambda} \text { where } u_{d, k} \text { are the eigenvalues of }-A_{d} \text { with } \\
& A_{d}=\left(\left(a_{d}\right)_{i j}\right)(i, j=1, \cdots, N), \quad\left(a_{d}\right)_{i j}=a_{j i}(i \neq j), \\
& a_{11}=-\mu_{1}, \quad a_{i i}=-\left(\lambda_{i-1}+\mu_{i}\right) \quad(i=2, \cdots, N), \\
& \sigma_{d, k}:=\left(\sum_{i=0}^{N-1}\left(Q_{d, 1}^{2}\left(u_{d, k}\right) \Delta_{i}\right)^{-1}\right.
\end{aligned}
$$

and $\left(Q_{d, 0}\left(u_{d, k}\right), \cdots, Q_{d, N-1}\left(u_{d, k}\right)\right)^{T}$ is the eigenvector of $-A_{d}$ corresponding to $u_{d, k}$, $Q_{d, 0}\left(u_{d, k}\right) \equiv 1(k=1, \cdots, N)$.

The quasidiffusion with speed measure $m_{d}$ is the birth- and death-process on

$$
\left\{0, m_{1}, m_{1}+m_{2}, \cdots, \sum_{1}^{N-1} m_{k}\right\} \quad \text { with } A_{d} \text { as its matrix of intensities. }
$$

\section{A sojourn times over positive levels.}

Assume $X$ is a quasidiffusion with speed measure $m$ and suppose $r_{0} \leqq 0$ and $0 \in E_{m}$. Moreover, choose $x, a, a^{\prime} \in E_{m}$ with $0<x \leqq a^{\prime} \leqq a \leqq r_{1}$ and put $G:=\left[a^{\prime}, l_{1}\right)$. Then, from (6), (7) and the strong Markov property of $X$ it follows for $\lambda<0$ :

$$
\begin{aligned}
& E_{x} \exp \left(\lambda \tau_{0}^{G}\right) \mathbf{1}_{\left\{\tau_{a}<\tau_{0}<\zeta\right\}}= \\
= & \left.\left(E_{x} \exp \left(\lambda \tau_{a}^{G}\right) \mathbf{1}_{\left\{\tau_{a}<\tau_{0}\right\}}\right)\left(E_{a} \exp \left(\lambda \tau_{d}^{G}\right)\right) \mathbf{1}_{\left\{\tau_{0}<\tau_{l_{1}}\right\}}\right) \\
= & \frac{\chi_{-}^{G, 0}(x, \lambda)}{\chi_{-}^{G, 0}(a, \lambda)} \cdot \frac{\chi_{+}^{G}(a, \lambda)}{\chi_{+}^{G}(0, \lambda)}=: H\left(a, x, a^{\prime}, \lambda\right)
\end{aligned}
$$

(Here $\tau^{G}$. is understood as the last integral in (4).)

Using $D_{m} D_{x} f+\lambda f=0$ on $G$ for $f=\varphi^{G}$ and $f=\psi^{G}$ it follows that $\varphi^{G}, \psi^{G}$ are linear combinations of $\varphi$ and $\psi$ on $G$. Now, from $\varphi^{G}(y, \lambda)=1, \psi^{G}(y, \lambda)=y$ on $\left[0, a^{\prime}\right]$ we obtain

$$
\begin{aligned}
& \chi_{+}^{G}(a, \lambda)=-\frac{\chi_{+}(a, \lambda)}{D^{-} \chi_{+}(a, \lambda)}, \\
& \chi_{+}^{G}(0, \lambda)=a^{\prime}-\frac{\chi_{+}\left(a^{\prime}, \lambda\right)}{D^{-} \chi_{+}\left(a^{\prime}, \lambda\right)},
\end{aligned}
$$


Sojourn times, excursions and spectral measures

$$
\begin{gathered}
\chi_{-}^{G, 0}\left(a^{\prime}, \lambda\right)=D^{-} \chi_{-}^{G, 0}\left(a^{\prime}, \lambda\right)\left[-\varphi(a, \lambda) \psi\left(a^{\prime}, \lambda\right)+\varphi\left(a^{\prime}, \lambda\right) \psi(a, \lambda)-\right. \\
\left.-a^{\prime}\left(D^{-} \varphi\left(a^{\prime}, \lambda\right) \psi(a, \lambda)-D^{-} \psi\left(a^{\prime}, \lambda\right) \varphi(a, \lambda)\right)\right] \\
\chi_{-}^{G, 0}(x, \lambda)=x \cdot D^{-} \chi_{-}^{G, 0}\left(a^{\prime}, \lambda\right) \quad\left(\lambda \in K_{0}\right) .
\end{gathered}
$$

Observe that

$$
h_{0}^{a,+}(\lambda):=\frac{\psi(a, \lambda)}{\varphi(a, \lambda)} \quad\left(\lambda \in K_{0}\right)
$$

is the characteristic function of the string

$$
\mathbf{1}_{[0, a]}(x) m(d x)+\infty \cdot \delta_{a}(d x)
$$

$\left(\delta_{a}\right.$ denotes the measure with unit mass concentrated at $a$ ) and that it holds

$$
\begin{aligned}
& \chi_{+}^{a}\left(a^{\prime}, \lambda\right)=\varphi\left(a^{\prime}, \lambda\right) \int_{a^{\prime}}^{a} \varphi^{-2}(s, \lambda) d s= \\
= & \varphi\left(a^{\prime}, \lambda\right)\left(\frac{\psi(a, \lambda)}{\varphi(a, \lambda)}-\frac{\psi\left(a^{\prime}, \lambda\right)}{\varphi\left(a^{\prime}, \lambda\right)}\right)=h_{0}^{a,+}(\lambda) \varphi\left(a^{\prime}, \lambda\right)-\psi\left(a^{\prime}, \lambda\right) .
\end{aligned}
$$

In the following we write $h$ and $h^{a}$ instead of $h_{0}^{+}$and $h_{0}^{a,+}$, respectively. Then we obtain for $\lambda<0$

$$
H\left(x, a, a^{\prime} \lambda\right)=\frac{x\left(h(\lambda)-h^{a}(\lambda)\right)}{\left.\left[\chi_{+}\left(a^{\prime}, \lambda\right)-a^{\prime} D^{-} \chi_{+}\left(a^{\prime}, \lambda\right)\right)\right]\left[\chi_{+}^{a}\left(a^{\prime}, \lambda\right)-a^{\prime} D^{-} \chi_{+}^{a}\left(a^{\prime}, \lambda\right)\right]}
$$

If $\lambda \uparrow 0$ it follows from (23)

$$
P_{x}\left(\tau_{a}<\tau_{0}<\zeta\right)=\frac{x}{a} \cdot \frac{l_{1}-a}{l_{1}} \text { with } \quad \frac{l_{1}-a}{l_{1}}=1 \quad \text { if } \quad l_{1}=\infty .
$$

Thus we have proved

Proposition 3.1. If $0<x \leqq a^{\prime} \leqq a \leqq r_{1}\left(x, a, a^{\prime} \in E_{m}\right)$, then for $\lambda<0$

$$
\begin{aligned}
& \phi\left(a, a^{\prime}, \lambda\right):=E_{x}\left[\exp \left(\lambda \int_{0}^{\tau} \mathbf{1}_{\left[a^{\prime}, l_{1}\right)}\left(X_{s}\right) d s\right) \mid \tau_{a}<\tau_{0}<\zeta\right]= \\
= & \mu^{-1} \cdot \frac{h(\lambda)-h^{a}(\lambda)}{\left(\chi_{+}\left(a^{\prime}, \lambda\right)-a^{\prime} D^{-} \chi_{+}\left(a^{\prime}, \lambda\right)\right)\left(\chi_{+}^{a}\left(a^{\prime}, \lambda\right)-a^{\prime} D^{-} \chi_{+}^{a}\left(a^{\prime}, \lambda\right)\right)} \\
= & \mu^{-1} \cdot \frac{h(\lambda)-h^{a}(\lambda)}{h^{G}(\lambda) h^{G, a}(\lambda)} \cdot D^{-} \chi_{+}\left(a^{\prime}, \lambda\right) D^{-} \chi_{+}^{a}\left(a^{\prime}, \lambda\right)
\end{aligned}
$$

with the notation $\mu:=a^{-1}-l_{1}^{-1}$.

(Recall that $h^{a}, h^{G}$, and $h^{G, a}$ are the characteristic functions of

$$
\mathbf{1}_{[0, \infty)} d m, \mathbf{1}_{[0, a]} d m+\infty \delta_{a} ; \mathbf{1}_{[0, \infty)} d m^{G} \text { and } \mathbf{1}_{[0, a]} d m^{G}+\infty \delta_{a}
$$

respectively.) 
Let $a^{\prime} \uparrow a\left(a^{\prime} \in E_{m}\right)$. Then, by using

$$
\begin{aligned}
& h(\lambda)-h^{a}(\lambda)=\frac{\chi_{+}(a, \lambda)}{\varphi(a, \lambda)}, \quad \chi_{+}^{a}\left(a^{\prime}, \lambda\right) \rightarrow 0 . \\
& D^{-} \chi_{+}^{a}\left(a^{\prime}, \lambda\right) \rightarrow-\varphi^{-1}(a, \lambda)\left(a^{\prime} \uparrow a\right) \text { and }(21)
\end{aligned}
$$

we obtain

$$
\begin{aligned}
& \phi(a, \lambda):=\lim _{a^{\prime} \uparrow^{a}} \phi\left(a, a^{\prime}, \lambda\right)=(a \mu)^{-1} \cdot \frac{\chi_{+}(a, \lambda)}{\chi_{+}(a, \lambda)-a D^{-} \chi_{+}(a, \lambda)}= \\
= & \mu^{-1} \cdot \int_{(0, \infty)} \frac{u}{u-\lambda} \sigma_{d}^{G}(d u)=\mu^{-1} \cdot \int_{0}^{\infty} e^{u \lambda} \vartheta_{d}^{G}(u) d u
\end{aligned}
$$

with

$$
G=[a, \infty), \quad \vartheta_{d}^{G}(u):=\int_{(0, \infty)} v e^{-u v} \sigma_{d}^{G}(d v)
$$

Because of

$$
\sigma_{d}^{G}((0, \infty))=\int_{0}^{\infty} \vartheta_{d}^{G}(u) d u=a^{-1}-l_{1}^{-1}=\mu<\infty \quad(\text { see }(19)),
$$

we can introduce a probability $\sigma$ on $(0, \infty)$ by

$$
\sigma(A):=\mu^{-1} \sigma_{d}^{G}(A) . \quad(A \in \mathfrak{B} \cap(0, \infty)) .
$$

Obviously, $\phi(a, \cdot)$ is the Laplace transform of a mixed exponential distribution with mixing measure $\sigma$. Thus we have shown the following

Proposition 3.2. If $0<x \leqq a \leqq r_{1}\left(x, a \in E_{m}\right)$, we have

$$
\begin{aligned}
& P_{x}\left(\int_{0}^{\tau_{0}} \mathbf{1}_{\left[a, l_{1}\right)}\left(X_{s}\right) d s \in d t \mid \tau_{a}<\tau_{0}<\zeta\right)= \\
= & d t \int_{(0, \infty)} u e^{-u t} \sigma(d u)=\mu^{-1} \vartheta_{d}^{G}(t) d t .
\end{aligned}
$$

Let $0<a<l_{1} \leqq \infty$ be fixed, $G=\left[a, l_{1}\right)$ and denote by $\mathfrak{M}_{a}$ the set of all strings $m$ from $\mathfrak{M}$ with $a \in E_{m^{G}}$. Then the mapping $m^{G} \rightarrow \sigma$ defined by Proposition 3.2. is a oneonto-one correspondence between the set of all strings of the form

$$
d m^{G}(x)=\mathbf{1}_{G}(x) d m(x)+\infty \delta_{l_{1}}(x) d x \quad\left(m \in \mathfrak{M}_{a}\right)
$$

and the set of probabilities $\sigma$ on $(0, \infty)$.

This is a consequence of M.G. Krein's inverse spectral theorem. (Indeed, given a probability $\sigma$ on $(0, \infty)$ define $\sigma_{1}:=\mu \sigma$ with $\mu=a^{-1}-l_{1}^{-1}$, supplement it by mass $l_{1}^{-1}$ at zero and choose, by Krein's theorem (see e.g. [2, 6, 10]) the uniquely determined string $n$ having $\sigma_{1}$ as its spectral measure. (12) and (13) imply $n(\{0\})=a, n([0, \infty))=$ $l_{1}$. Take the dual string $n_{d}$, which turns out to be of the form $m^{G}$ with the desired properties.) 


\section{Examples:}

1. Consider the standard Wiener process $W$, it is a quasidiffusion on $R$ with speed measure $m(x)=2 x(x \in R)$, and choose $a>0$. Then, for the characteristic function $h_{0, d}^{G,+}$ of the dual string of $\mathbf{1}_{G}(x) d m(x)(G=[a, \infty))$ we get

$$
h_{0, d}^{G,+}(\lambda)=-\frac{2 a \lambda+\sqrt{-2 \lambda}}{\lambda+2 a^{2} \lambda^{2}} \quad\left(\lambda \in K_{0}\right)
$$

and for the normalized measure $\sigma$ we obtain

$$
\sigma(d u)=\frac{2 a}{\pi \sqrt{2 u}}-\frac{1}{1+2 u a^{2}} d u \quad(u>0) .
$$

Then it follows

$$
P_{x}\left(\int_{0}^{\tau_{0}} \mathbf{1}_{[a, \infty)}\left(W_{s}\right) d s>t \mid \tau_{a}<\tau_{0}\right)=\frac{2 a}{\pi} \int_{0}^{\infty} \frac{e^{-\frac{v^{2}}{2} t}}{1+v^{2} a^{2}} d v \quad(x \in(0, a]) .
$$

2. Let $X$ be a birth- and death-process with finite state space $\{0,1, \cdots, N\}$ and intensities

$$
\lambda_{i}>0 \quad(i=0, \cdots, N-1) \quad \mu_{i}>0 \quad(i=1, \cdots, N)
$$

Then, starting at 1 the hitting time $\tau_{0}=\inf \left\{t>0 / X_{t}=0\right\}$ has a mixed exponential distribution

$$
P_{1}\left(\tau_{0} \in d t / X_{0}=1\right)=\sum_{k=1}^{N} W_{k} \exp \left(-W_{k} t\right) \sigma_{k} d t
$$

where $W_{k}(k=1, \cdots, N)$ are the eigenvalues of the matrix $-A_{d}$ (see above) and

$$
\sigma_{k}:=\sigma_{d, k} \cdot\left(\sum_{1}^{N} \sigma_{d, j}\right)^{-1} \quad(k=1, \cdots, N)
$$

with $\sigma_{d, k}$ given by (22).

\section{Excursions, local time and spectral measure.}

\subsection{Excursions of the Wiener process.}

Assume $W=\left(W_{t}, \mathscr{F}_{t}^{W}, P_{x}\right)$ is a standard Wiener process. In this point we consider $W$ under the measure $P_{0}$ only, i.e. $W$ starts at zero. The open set $[0, \infty) \backslash\left\{t \geqq 0 / W_{t}\right.$ $=0\}$ is the union of its components $\left(a_{n}^{W}, b_{n}^{W}\right)(n \geqq 1)$. For every $n \geqq 1$ the process $U_{n}^{W}$ given by

$$
U_{n}^{W}(t)=W_{t+a_{n}} \quad\left(0 \leqq t \leqq b_{n}^{W}-a_{n}^{W}\right), \quad=0 \quad\left(t \geqq b_{n}^{W}-a_{n}^{W}\right)
$$

is called an excursion of $W$. We introduce the length $T_{n}^{W}$ and the peak $V_{n}^{W}$ of the excursion $U_{n}^{W}$ by defining 


$$
T_{n}^{W}:=b_{n}^{W}-a_{n}^{W}, V_{n}^{W}:=\max _{t \geqslant 0} U_{n}^{W}(t) \operatorname{sgn} U_{n}^{W}\left(\frac{b_{n}^{W}+a_{n}^{W}}{2}\right) .
$$

If $n$ is not specified we write $U^{W}, V^{W}, T^{W}$ only. K. Ito proved ([4], see also [14]) that the points $\left(l^{W}\left(a_{n}^{W}, 0\right), U_{n}^{W}\right)$ are the atoms of a Poisson measure $Q$ on $((0, \infty) \times U$, $(\mathfrak{B}(0, \infty)) \otimes \mathcal{U})$ where $(U, \mathcal{U})$ denotes the space of all excursions. Moreover, he showed that the $\sigma$-finite measure $n$ given by $n(\Lambda):=E Q(\Lambda)$ satisfies

$$
n(d t, d f)=d t \nu^{W}(d f)
$$

where $\nu^{W}$ is a measure on $(U, \mathcal{U})$, called the Ito excursion law of $W$ at zero. It holds

$$
\nu^{W}\left(V^{W} \in d x\right)=x^{-2} d x \quad(x \in R \backslash\{0\})
$$

Before using these properties we shall introduce some notations. If $E$ is a Borel subset of $R \backslash(-\varepsilon, \varepsilon)$ for some $\varepsilon>0$ define $N_{t}^{W}(E)$ to be the number of all excursions $U_{n}^{W}$ with $V_{n}^{W} \in E$ and $l^{W}\left(a_{n}, 0\right) \leqslant t(t \geqslant 0)$. Thus $N_{t}(E)$ counts the excursions of $W$ from zero with peak in $E$ which are finished before

$$
L^{W}(t, 0):=\inf \left\{s>0 \mid l^{W}(s, 0)>t\right\} \quad(t \geqslant 0)
$$

or, equivalently, up to the moment, when the local time $l^{W}(\cdot, 0)$ equals $t$. From the mentioned Ito's results it easily follows

Lemma 4.1. Let $E_{1}, E_{2}$ be disjoint Borel subsets of

$$
\{x \in R|| x \mid>\varepsilon\} \text { for some } \varepsilon>0 .
$$

Then $N^{W}\left(E_{k}\right)$ are mutually independent Poisson processes with intensities

$$
\nu^{W}\left(V^{W} \in E_{k}\right) \quad(k=1,2) .
$$

Denote by $U_{k_{j}}^{V}(j \geqslant 1)$ the $j$-th excursion of $W$ from 0 with peak in $E_{k}$ and let $s(d x)$ be $a$-finite measure on $R$. Then for every $A \in \mathfrak{B}$ the variables

$$
y_{k_{1}}(A):=\int_{A}\left(l^{W}\left(b_{k_{j}}^{W}, x\right)-l^{W}\left(a_{k_{j}}^{W}, x\right)\right) s(d x) \quad(k=1,2 ; j \geqq 1)
$$

are mutually independent, identically distributed and independent of $N^{W}\left(E_{k}\right)(k=1,2)$.

Assume $a, l_{0}, l_{1}$ to be real numbers with $l_{0}<0<a<l_{1}$ and put $E_{1}=\left[a, l_{1}\right), E_{2}=R \backslash\left(l_{0}, l_{1}\right)$. Then the preceding lemma implies

Corollary 4.2. The number $N_{t}^{W}\left(E_{1}\right)$ of excursions of $W$ with peaks in $\left[a, l_{1}\right)$ which occur before $L^{W}(t, 0)$ form a Poisson process with intensity $\mu=a^{-1}-l_{1}^{-1}$ which is independent of

$$
l^{W}\left(\zeta^{W}, 0\right)=\inf \left\{t>0 \mid N_{t}^{W}\left(E_{2}\right) \geqq 1\right\},
$$

and this variable is exponentially distributed with the parameter $\kappa:=l_{1}^{-1}-l_{0}^{-1}$. 


\subsection{Excursions of quasidiffusions.}

Introducing the excursions of a quasidiffusion $X$ from 0 we have to take into consideration the role of the gaps of $E_{m}$ and the killing time $\zeta$. Let $X$ be a quasidiffusion with speed measure $m$ as constructed in 2.1. above, we also assume here that $X$ starts at zero. Put

$$
a_{n}:=S_{a_{n}^{W}}, \quad b_{n}:=S_{b_{n}^{W}} \quad(n \in N)
$$

It may happen that $a_{n}=b_{n}$ (this means the excursion $U_{n}^{W}$ does not meet $E_{m} \backslash\{0\}$ ) or that $b_{n}=\infty$ (i.e. $\mathrm{W}$ hits $l_{0}$ or $l_{1}$ before $b_{n}^{W}$ ). Therefore we define

$$
\begin{aligned}
& \mathscr{N}:=\left\{n \geqq 1 \mid a_{n}<b_{n}<\zeta\right\} \quad \text { and put } \\
& U_{n}(t):=X_{t+a_{n}} \quad\left(0 \leqq t<b_{n}-a_{n}\right), \quad=0\left(t \geqq b_{n}-a_{n}\right)(n \in \mathscr{M})
\end{aligned}
$$

The processes $U_{n}(n \in \mathscr{N})$ are called the excursions of $X$ from 0 . This notation is justified by the following properties:

a) $U_{n}(t) \neq 0$ for $t \in\left(a_{n}, b_{n}\right) \quad(n \in \mathcal{N})$

b) If $\tau:=\sup \left\{t<\zeta \mid X_{t}=0\right\}$ and $t \in[0, \tau)$ with $X_{t} \neq 0$, then $t \in\left(a_{n}, b_{n}\right)$ for some $n \in \mathscr{N}$.

If $V_{n}^{W}>0$ then $V_{n}=\left(V_{n}^{W}\right)_{-}=\sup \left\{y<V_{n}^{W} \mid y \in E_{m}\right\}$. Now choose $a>0, a \in E_{m}$ and define $N_{t}(a)$ to be the number of all excursions $U_{n}(n \in \mathscr{I})$ such that $V_{n} \in\left(a, l_{1}\right)$ and $l\left(a_{n}, 0\right) \leq t$. Then (recall the definition of $\left.\eta\right) N_{t}(a)$ counts the number of excursions of $X$ from 0 over the level $a$ which occur before $L(t, 0) \wedge \zeta$ where $L(\cdot, 0)$ denotes the inverse local time:

$$
L(t, 0):=\inf \{s>0 \mid l(s, 0)>t\} \quad(t \geqslant 0) .
$$

By virtue of $l(\zeta, 0)=l^{W}\left(\zeta^{W}, 0\right)$ we have

$$
N_{t}(a)=N_{t \wedge l^{W}}^{W}\left(\zeta^{W}, 0\right)\left(\left[a, l_{1}\right)\right) \quad(t \geqslant 0) .
$$

Thus from Corollary 4.2. and the second half of Lemma 4.1. with $s(d x)=m(d x)$ we conclude

Proposition 4.3. For every $a \in E_{m}$, $a>0$, the process $N(a)$ forms a Poisson process with intensity $\mu=a^{-1}-l_{1}^{-1}$ which is stopped at the random time $l(\zeta, 0)$. The variable $l(\zeta, 0)$ is independent of $N(a)$ and exponentially distributed with the parameter $\kappa=l_{1}^{-1}-l_{0}^{-1}$.

For every $A \in \mathfrak{B}$ the variables

$$
\begin{aligned}
Y_{n}(A): & =\int_{A}\left(l^{W}\left(b_{n}^{W}, x\right)-l^{W}\left(a_{n}^{W}, x\right)\right) m(d x)= \\
& =\int_{A}\left(l\left(b_{n}, x\right)-l\left(a_{n}, x\right)\right) m(d x)=\int_{a_{n}}^{b_{n}} \mathbf{1}_{A}\left(X_{s}\right) d s \quad(n \in \mathcal{M})
\end{aligned}
$$

are mutually independent, independent of $N(a)$ and identically distributed.

Choose an $a^{\prime} \in(0, a) \cap E_{m}$ and put 


$$
Y_{n}\left(a^{\prime}\right):=Y_{n}\left(\left[a^{\prime}, l_{1}\right)\right) \quad(n \in \mathscr{N}) .
$$

The strong Markov property of $X$ implies that the Laplace transform

$$
E_{0}\left(\exp \left(\lambda Y_{n}\left(a^{\prime}\right)\right) \mid V_{n} \geqslant a\right)
$$

of $Y_{n}\left(a^{\prime}\right)$ under $\left\{V_{n} \geqslant a\right\}$ equals

$E_{a^{\prime}}\left(\exp \left(\lambda \int_{0}^{\tau_{0}} \mathbf{1}_{\left[a^{\prime}, l_{1}\right)}\left(X_{s}\right) d s\right) \mid \tau_{a}<\tau_{0}<\zeta\right)$, i.e. it is given by $\Phi\left(a, a^{\prime}, \lambda\right)$ from formula (24) $(n \in \mathscr{M}, \lambda<0)$. Put

$$
P_{t}\left(a, a^{\prime}\right):=\sum Y_{n}\left(a^{\prime}\right) \quad(t \geqslant 0)
$$

where the sum is going over all $n \in \mathscr{N}$ such that $l\left(a_{n}, 0\right) \leqslant t$ and $V_{n} \in\left[a, l_{1}\right)$, and define

$$
P\left(a, a^{\prime}\right):=\left(P_{t}\left(a, a^{\prime}\right)\right)_{t \geqslant 0} \text {. }
$$

The variable $P_{t}\left(a, a^{\prime}\right)$ is equal to the time which $X$ spends over $a^{\prime}$ during excursions of $X$ from zero to $a$ occuring before $L(t, 0) \wedge \zeta$, i.e. before the local time $l(\cdot, 0)$ equals $t \wedge l(\zeta, 0)$.

From the Proposition 4.3. and the remarks after this proposition it follows now

Theorem 4.4. Assume $0<a^{\prime}<a$ with $a, a^{\prime} \in E_{m}$. Then the process $P\left(a, a^{\prime}\right)$ is an increasing compounded Poisson process with intensity $\mu=a^{-1}-l_{1}^{-1}$ stopped at the random time $l(\zeta, 0)$ which is independent of $P\left(a, a^{\prime}\right)$ and exponentially distributed with the parameter $\kappa=l_{1}^{-1}-l_{0}^{-1}$.

In particular

$$
\begin{aligned}
& \Phi_{t}\left(a, a^{\prime}, \lambda\right):=E_{0}\left(\exp \left(\lambda P_{t}\left(a, a^{\prime}\right)\right) \mid l(\zeta, 0)>t\right)= \\
= & \exp \left(-\mu t\left(1-\Phi\left(a, a^{\prime}, \lambda\right)\right)\right) \quad(t \geqslant 0, \lambda<0)
\end{aligned}
$$

with $\Phi\left(a, a^{\prime}, \lambda\right)$ as given in (24).

Proposition 4.5. The process $P(a, 0):=\left(P_{t}(a, 0)\right)_{t \geq 0}$ is an increasing compounded Poisson process with intensity $\mu$ stopped at $l(\zeta, 0)$ which is independent of $P(a, 0)$ and exponentially distributed with the parameter $\kappa$. It holds

$$
\begin{aligned}
& E_{p}\left(\exp \left(\lambda P_{t}(a, 0)\right) \mid l(\zeta, 0)>t\right)=\exp \left(-t\left[\left(\frac{1}{a}-\frac{1}{h^{a}(\lambda)}\right)-\left(\frac{1}{l_{1}}-\frac{1}{h(\lambda)}\right)\right]\right) \\
= & \exp \left(-t\left(\int_{0}^{\infty}\left(1-e^{\lambda u}\right)\left(\vartheta_{d}(u)-\vartheta_{d}^{(a)}(u)\right) d u\right) \quad(t \geqq 0, \lambda<0),\right.
\end{aligned}
$$

where

$$
\vartheta_{d}^{(a)}(u)=\int_{(0, \infty)} v e^{-v u} \sigma_{d}^{(a)}(d v), \quad(u>0)
$$

and $\sigma_{d}^{(a)}$ denotes the spectral measure of the string dual to

$$
\mathbf{1}_{[0, a)}(x) m(d x)+\delta_{a}(d x) \cdot \infty .
$$


Note that the integral

$$
\int_{0}^{\infty}\left(\vartheta_{d}(u)-\vartheta_{d}^{(a)}(u)\right) d u=\lim _{\lambda \downarrow-\infty} \int_{0}^{\infty}\left(1-e^{\lambda u}\right)\left(\vartheta_{d}(u)-\vartheta_{d}^{(a)}(u)\right) d u
$$

equals $\mu$ (this also follows from

$$
\frac{1}{h^{a}(\lambda)}-\frac{1}{h(\lambda)}=\frac{\varphi(a, \lambda)}{\psi(a, \lambda)}-\lim _{x_{\uparrow} l_{1}} \frac{\varphi(x, \lambda)}{\psi(x, \lambda)}=\int_{a}^{l_{1}} \psi^{-2}(s \lambda) d s \rightarrow 0
$$

for $\lambda \downarrow-\infty)$ but the integrals over $\vartheta_{d}$ and over $\vartheta_{d}^{(a)}$ must not be finite.

(We supposed $a^{\prime} \downarrow 0, a^{\prime} \in E_{m}$, which demands $0_{+}=0$. However the results are valid for $0_{+}>0$, too. This becomes obvious by using $\chi_{+}\left(0_{+}, \lambda\right)-0_{+} \cdot D^{-} \chi_{+}\left(0_{+}, \lambda\right)=h(\lambda)$. ) In particular, the preceding Proposition implies that the distribution of the length

$$
T_{n}=y_{n}(0)=\int_{a_{n}}^{b_{n}} \mathbf{1}_{\left[0, l_{1}\right)}\left(x_{s}\right) d s
$$

under $\left\{V_{n} \geqq a\right\}$ is given by

$$
P_{0}\left(T_{n} \in d u \mid V_{n} \geqslant a\right)=\mu^{-1}\left(\vartheta_{d}(u)-\vartheta_{d}^{(a)}(u)\right) d u \quad(u \geqslant 0)
$$

With these remarks Proposition 4.5. provides a connection between Ito's excursion law of $X$ and the spectral measures of two dual strings:

Corollary 4.6. Assume 0 is a recurrent state, i.e. $l_{1}=-l_{0}=\infty$ and therefore $\zeta=\infty$. Denote by $\nu$ the Ito excursion law of $X$ at zero, by $T$ the length and by $V$ the peak of the excursion $U$ of $X$ from 0 . Then it holds

$$
\begin{aligned}
& \nu(V \geqslant a)=\nu^{W}\left(V^{W} \geqslant a\right)=a^{-1} \\
& \nu(T \in d u, V \geqslant a)=\nu(V \geqslant a) P_{0}(T \in d u \mid V \geqslant a) \\
& =\left(\vartheta_{d}(u)-\vartheta_{d}^{(a)}(u)\right) d u \\
& \nu(T \in d u)=\vartheta_{d}(u) d u \text { and } \\
& \int_{0}^{\infty}\left(1-e^{\lambda t}\right) \nu(T \in d t, V \geqslant a)=\frac{1}{h(\lambda)}-\left(\frac{1}{h^{a}(\lambda)}-\frac{1}{a}\right) .
\end{aligned}
$$

In particular (30) implies now

$$
\int_{0}^{\infty} e^{\lambda t} \nu(T \in d t, V \geqslant a)=\frac{1}{h^{a}(\lambda)}-\frac{1}{h(\lambda)}=\int_{a}^{\infty} \psi^{-2}(x, \lambda) d x \quad\left(a \in E_{m}\right)
$$

This formula extends a result for the standard Wiener process $(m(x)=2 x, x \in R)$ for which $\psi(x, \lambda)=\frac{\sinh \sqrt{-2 \lambda x}}{\sqrt{-2 \lambda}}$ holds (see Williams [14], p. 99). In this case we get

$$
\nu(T \in d t, V \geqslant a)=\left(\left(2 \pi t^{3}\right)^{-1 / 2}-\frac{1}{a} \sum_{k=1}^{\infty} \frac{k^{2} \pi^{2}}{a^{2}} \exp \left(-\frac{k^{2} \pi^{2}}{2 a^{2}}\right)\right) d t \quad(t>0, a>0) .
$$


Now let us consider the second special case: $a^{\prime} \uparrow a$ where $a>0, a \in E_{m}$ is fixed. From (25) and (27) it follows

$$
\begin{aligned}
& \Phi_{t}(a, \lambda):=\lim _{u^{\prime} \uparrow^{a}} \Phi_{t}\left(a, a^{\prime}, \lambda\right)=\exp (-\mu t(1-\Phi(a, \lambda)))= \\
= & \exp \left(-\mu t\left(1-\int_{(0, \infty)} \frac{u}{u-\lambda} \sigma(d u)\right)\right)= \\
= & \exp \left(-t \int_{0}^{\infty}\left(1-e^{u \lambda}\right) \vartheta_{d}^{G}(u) d u\right) \quad(\lambda<0),
\end{aligned}
$$

where $G=\left[a, l_{1}\right), \sigma=\mu^{-1} \sigma_{d}^{G}, \vartheta_{d}^{G}(u)=\int_{(0, \infty)} v e^{-u v} \sigma_{d}^{G}(d v)$ with $\int_{0}^{\infty} \vartheta_{d}^{G}(u) d u=\mu$ and $\sigma_{d}^{G}$ is the spectral measure of the string dual to $m^{G}$. Then we have shown

Proposition 4.8. The process $P(a, a)=\left(P_{t}(a, a)\right)_{t \geq 0}$ is an increasing compounded Poisson process with intensity $\mu$ stopped at the random time $l(\zeta, 0)$ which is independent of $P(a, a)$ and exponentially distributed with the parameter $\kappa$. It holds

$$
E_{0}\left(\exp \left(\lambda P_{t}(a, a)\right) \mid l(\zeta, 0)>t\right)=\Phi_{t}(a, \lambda)
$$

where $\Phi_{t}(a, \lambda)$ is given by $(32)$.

Note that

$$
P_{t}(a, a)=\int_{0}^{l(t, 0) \zeta} \mathbf{1}_{\left[a, l_{1}\right)}\left(X_{s}\right) d s \quad(t \geqq 0)
$$

(These properties also hold, if $a$ is not an accumulation point of $E_{m}$ from the left.)

In both particular cases $a^{\prime} \downarrow 0$ and $a^{\prime} \uparrow a$ we have explicit representations of the Lévy-measure of the increasing compounded Poisson process $P=\left(P_{t}\right)_{t \geqq 0}$ by spectral measures of some corresponding dual strings. Moreover, in these cases the distribution of $P_{t}$ under $\{l(\zeta, 0)>t\}$ belongs to the so-called Bondesson-class of distributions (see [1]). (It should be remarked, that every distribution of the Bondesson-class occur if $m$ runs through all strings, this is also a consequence of M.G. Krein's theorem mentioned above.)

It remains an oepn problem to derive similar statements for the general case given in (27).

\subsection{Local times and spectral measures.}

We consider (32) again and let $a \downarrow 0$. Then, from (25) we have

$$
\begin{aligned}
& \Phi_{t}(0,0, \lambda):=E_{0}\left(\exp \left(\lambda \int_{0}^{L(t, 0)} \mathbf{1}_{(0, \infty)}\left(X_{s}\right) d s\right) \mid l(\zeta, 0)>t\right)= \\
= & \lim _{a \downarrow 0} \Phi_{t}(a, a, \lambda)=\lim _{a_{\downarrow} 0} \exp \left[t\left(l_{1}^{-1}+\frac{D^{-} \chi_{+}(a, \lambda)}{\chi_{+}(a, \lambda)-a D^{-} \chi_{+}(a, \lambda)}\right)\right]= \\
= & \exp \left[t\left(l_{1}^{-1}+\frac{D^{+} \chi_{+}(0, \lambda)}{\chi_{+}(0, \lambda)}\right)\right]=\exp \left[t\left(\frac{1}{l_{1}}-\frac{1}{h(\lambda)}-\lambda m_{0}\right)\right] \quad(\lambda<0, t \geqq 0)
\end{aligned}
$$


(Note that $\left(\frac{1}{h(\lambda)}+\lambda m_{0}\right)^{-1}$ is the characteristic function of the string $\mathbf{1}_{(0, \infty)}(x) d m(x)$, see (9).)

From $(2)$ and $l(L(t, 0), 0)=t$ on $\{l(\zeta, 0)>t\}$ it follows

$$
E_{0}\left(\exp \left(\lambda \int_{0}^{L(t, 0)} \mathbf{1}_{\{0\}}\left(X_{s}\right) d s \mid l(\zeta, 0)>t\right)=\exp \left(\lambda t m_{0}\right) \quad(\lambda<0, t \geqq 0)\right.
$$

Define $A_{1}:=\left(l_{0}, 0\right), A_{2}:=\left[0, l_{1}\right], P_{t}\left(A_{i}\right):=\int_{0}^{L(t, 0) \wedge \zeta} \mathbf{1}_{A_{i}}\left(X_{s}\right) d s(t \geqq 0, i=1,2)$. Then we have shown

Proposition 4.9. The two processes $P\left(A_{i}\right):=\left(P_{t}\left(A_{i}\right)\right)_{t \geqq 0}(i=1,2)$ are independent increasing processes with independent stationary increments killed at the random time $l(\zeta, 0)$ which is independent of $P\left(A_{i}\right)(i=1,2)$ and exponentially distributed with the parameter $\kappa$. Furthermore it holds

$$
\begin{aligned}
& E_{0}\left(\exp \left(\lambda P_{t}\left(A_{i}\right)\right) \mid l(\zeta, 0)>t\right)= \\
= & \exp \left[-t\left(\frac{1}{l_{0}}+\frac{1}{h_{0}^{-}(\lambda)}\right)\right] \quad \text { for } i=1, \\
= & \exp \left[-t\left(-\frac{1}{l_{1}}+\frac{1}{h_{0}^{+}(\lambda)}\right)\right] \quad \text { for } i=2 \quad(\lambda<0) .
\end{aligned}
$$

Applying (18) we obtain

$$
\begin{aligned}
& \frac{1}{l_{0}}+\frac{1}{h_{0}^{-}(\lambda)}=\int_{(0, \infty)}\left(1-e^{\lambda u}\right) \vartheta_{0, d}^{-}(u) d u \\
& -\frac{1}{l_{1}}+\frac{1}{h_{0}^{+}(\lambda)}=\int_{(0, \infty)}\left(1-e^{\lambda u}\right) \vartheta_{p, d}^{+}(u) d u+\lambda m_{0}
\end{aligned}
$$

with

$$
\vartheta_{0, d}^{ \pm}(u)=\int_{(0, \infty)} v e^{-u v} \sigma_{0, d}^{ \pm}(d v) \quad(u>0)
$$

where $\sigma_{0, d}^{+}$and $\sigma_{0, d}^{-}$denote the spectral measure of the string dual to $\mathbf{1}_{(0, \infty)} m_{0}^{+}$and $m_{0}^{-}$respectively. In particular we have

$$
\begin{aligned}
& E_{0}(\exp (\lambda L(t, 0)) \mid l(\zeta, 0)>t)= \\
= & \exp \left[-t\left(\lambda m_{0}+\int_{(0, \infty)}\left(1-e^{\lambda t t}\right) \vartheta_{0, d}(u) d u\right] \quad(\lambda<0)\right.
\end{aligned}
$$

with $\vartheta_{0, d}:=\zeta_{0, d}^{+}+\vartheta_{0, d}^{-}$.

Proposition 4.9. was proved for $r_{0}=0$ and $l_{1}=\infty$ in [9], see also [5] for the case of diffusions. Define

$$
G(\varepsilon):=\int_{\varepsilon}^{\infty} \vartheta_{0, d}(u) d u=\int_{(0, \infty)} e^{-v \varepsilon} \sigma_{0, d}(d v)
$$

with $\sigma_{0, d}:=\sigma_{0, d}^{+}+\sigma_{d, 0}^{-}$. 
The following proposition generalizes a result for diffusions from [5], Chapter 6.3.

Proposition 4.10. Assume $m_{0}=0,0 \in E_{m}$ and $l_{0}=\infty, l_{1}=-\infty$. Then it holds

$$
l(t, 0)=\lim _{\varepsilon \downarrow 0} \frac{\text { number of all excursions of } X \text { from } 0 \text { before } t \text { with length } \geqq \varepsilon}{G(\varepsilon)}
$$

Proof. The proof is similar to those for the Wiener process ([5], Chapter 2.2). We sketch it only. Firstly observe that $\lim _{\varepsilon \neq 0} G(\varepsilon)=\infty$. This comes from the assumption $m_{0}=0,0 \in E_{m}$ which implise that 0 is a point of accumulation of $E_{m}$. Therefore at least one of the functions $\vartheta_{0, d}^{+}$is not integrable. Furthermore we have $\zeta=\infty$ a.s. because of $\left|l_{i}\right|=\infty(i=0,1)$. We know from Proposition 4.9. that $(L(t, 0))_{t \geq 0}$ is an increasing process with independent stationary increments having the Lévy measure $\vartheta_{0, d}(u) d u(u>0)$. Thus by a strong law of large numbers it follows

$\lim G_{(\varepsilon)}^{-1}$. (Number of all jumps of $L(\cdot, 0)$ with magnitude $\geqq \varepsilon$ up to $\left.s\right)=s$ a.s.

Recall that the jumps of $L(\cdot, 0)$ up to $s$ correspond to the flat stretches of $l(\cdot, 0)$ up to $L(s, 0)$, i.e. to the excursions of $X$ from 0 up to $L(s, 0)$. Applying this, substituting $s=l(t, 0)$ and noting that $L(l(t, 0))-t$ is finite by virtue of $\lim _{u \rightarrow \infty} l(u, 0)=\infty$ we get the assertion.

It should be remarked that by applying Tauberian type theorems the limit behaviour of $G(\varepsilon)$ for $\varepsilon \downarrow 0$ can be obtained from the limits of

$$
\int_{0}^{\infty} e^{\lambda \varepsilon} G(\varepsilon) d \varepsilon=-\frac{1}{\lambda}\left(\frac{1}{h_{0}^{+}(\lambda)}+\frac{1}{h_{0}^{-}(\lambda)}\right) \quad \text { for } \quad \lambda \downarrow \infty .
$$

For instance, for the diffusion on $[0, \infty)$ with speed measure $m(x)=x^{\gamma}(x \geqq 0)$ (see example 1. in 2.3) we have

$$
G(\varepsilon) \sim \frac{1}{\Gamma\left(\frac{1}{\gamma+1}\right)}(\gamma+1)^{\frac{\gamma-1}{\gamma+1}} r^{\frac{1}{\gamma+1}} \varepsilon^{-\frac{1}{\gamma+1}} \quad(\varepsilon \downarrow 0)
$$

and for the example 2 from 2.3 we obtain

$$
G(\varepsilon) \sim \frac{1}{\Gamma\left(\frac{1}{2}\right)} \varepsilon^{-\frac{1}{2} \frac{1+c \tanh v}{c+\tanh v}}=\frac{1}{\sqrt{\pi \varepsilon}} \frac{1+c \tanh v}{c+\tanh v} \quad(\varepsilon \downarrow 0) .
$$

Sektion Mathematik

HUMBOLDT-UNIVERSITÄT ZU BERLIN

DDR-1086 BeRLIN, P.O.B. 1297 


\section{References}

[1] L. Bondesson, Classes of infinitely divisible distributions and densities, Z. Wahrscheinlichkeitstheorie verw. Gebiete, 57 (1981), 39-71.

[2] H. Dym and H. P. McKean, Gaussian processes, function-theory and the inverse spectral theorem, New York, Academic Press (1976).

[ 3 ] J. Groh, Über eine Klasse eindimensionaler Markov-processe, Math. Nachr., 65 (1976), 125-136.

[4] K. Ito, Poisson Point Processes attached to Markov Processes, Proc. 6th Berkeley Symp. Math. Statist. Prob., 3 (1971), 225-240.

[ 5 ] K. Ito, and H. P. McKean, Diffusion Processes and their Sample Paths, 2nd Printing, Springer, Berlin (1974).

[6] I. S. Kac, and M. G. Krein, On the spectral functions of the string, Amer. Math. Soc. Transl., (2) 103 (1974), 19-102.

[ 7 ] J. Kent, Eigenvalue expansion for diffusion hitting times, Z. Wahrscheinlichkeitstheorie verw. Geb., 52 (1980), 309-319.

[8] J. Kent, The spectral decomposition of a diffusion hitting time, Ann. Prob., 10, (1982) 207-219.

[9] F. B. Knight, Characterization of the Lévy measures of inverse local times of gap diffusion, Progress in Prob. Statist. 1, Birkhäuser, Boston, Mass. 1981.

[10] S. Kotani and S. Watanabe, Krein's spectral theory of strings and generalized diffusion processes, Lecture Notes of Mathematics Vol. 923, (1981) 235-259.

[11] U. Küchler, Some Asymptotic Properties of the Transition Densities of One-Dimensional Quasidiffusion, Publ. RIMS, Kyoto-University, 16 (1980), 245-268.

[12] P. Mandl, Analytical treatment of one-dimensional Markov processes, Academic Prague and Springer Verlag, Berlin (1968).

[13] C. Stone, Limit theorems for random walks, birth and death processes and diffusion processes, III. J. Math., 7 (1963), 638-660.

[14] D. Williams, Diffusions, Markov Processes and Martingales Vol. 1, J. Wiley and Sons, London (1979). 